\title{
Analysis and approximations for crossing two nearby spin resonances
}

\author{
V.H. Ranjbar
}

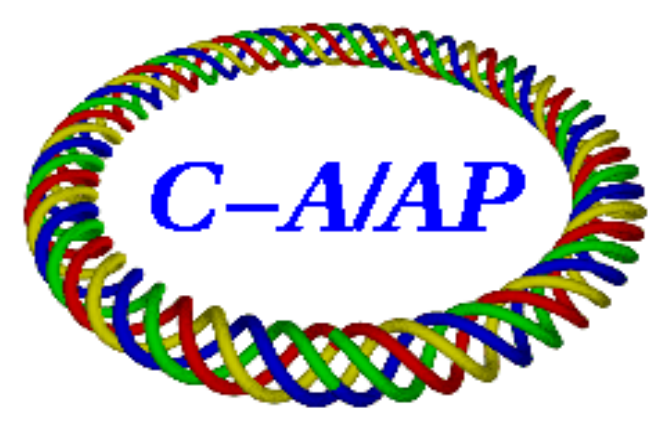

\section{Collider-Accelerator Department Brookhaven National Laboratory Upton, NY 11973}

Notice: This document has been authorized by employees of Brookhaven Science Associates, LLC under Contract No. DE-AC02-98CH10886 with the U.S. Department of Energy. The United States Government retains a nonexclusive, paid-up, irrevocable, world-wide license to publish or reproduce the published form of this document, or allow others to do so, for United States Government purposes. 


\title{
Analysis and Approximations for Crossing two nearby Spin Resonances
}

\author{
V. H. Ranjbar \\ Brookhaven National Lab, Upton NY 11973
}

(Dated: January 7, 2014)

\begin{abstract}
Solutions to the T-BMT spin equation have to date been confined to the single resonance crossing. However in reality most cases of interest concern the overlapping of several resonances. To date there has been several serious studies of this problem however a good analytical solution or even approximation has eluded the community. We show that the T-BMT equation can be transformed into a Hill's like equation. In this representation it can be shown that while the single resonance crossing represents the solution to the Parabolic Cylinder equation the overlapping case becomes a parametric type of resonance. We present possible approximations for both the non-accelerating case and accelerating case.
\end{abstract}

PACS numbers:

\section{INTRODUCTION}

The T-BMT equation has been much studied in various branches of physics. In the accelerator physics community it is used to model the spin dynamics which particles undergo in a beam-line. A well developed theoretical apparatus has been developed to handle the various spin depolarizing resonances. This is mostly based on our understanding of solutions to the single resonance model via Foissart-Stora formula [1] for accelerating particles and harmonic oscillator for the stationary case.

However in most cases the presence of nearby spin resonances cast doubt on the veracity of our understanding so an approach to handle the interference of nearby spin resonances has been long sought after. To date there have been several efforts made to understand the effect of nearby resonances. An approach base on classification of resonance proximity and strength was developed by S.Y. Lee [2] and S. Tepikian a number years ago. However this effort couldn't explain the case when resonances were nearly overlapping and accelerating. Later S. Mane [3] developed and approximation based on the first order Magnus expansion and applying a modified resonance strength add-hock to the Froissart-Stora formula with some limited success.

In this paper we present an approach which cast the problem into a form accelerator physics are fairly comfortable with and permit a clearer understanding of the dynamics driving the spin evolution during multiple resonance crossings.

\section{T-BMT IN SPINNOR FORM}

The dynamics of the spin vector of a charged particle is determined by the interaction of the magnetic moment with the surrounding magnetic field. In the particle's rest frame this is described simply by,

$$
\frac{d \vec{S}}{d t}=\vec{\mu} \times \vec{B}
$$

Here $\vec{S}$ is the spin vector of a particle and $\vec{\mu}$ is the mag- netic moment. If we transform to the laboratory frame then Eq. (1) becomes the Thomas-BMT (Bargmann, Michel, and Telegdi ) Equation,

$$
\begin{array}{r}
\frac{d \vec{S}}{d t}=\frac{e}{\gamma m} \vec{S} \times \\
\left((1+G \gamma) \vec{B}_{\perp}+(1+G) \vec{B}_{\|}+\left(G \gamma+\frac{\gamma}{\gamma+1}\right) \frac{\vec{E} \times \vec{\beta}}{c}\right) .
\end{array}
$$

$\vec{S}$ is the spin vector of a particle in the rest frame, and $\vec{B}_{\perp}$ and $\vec{B}_{\|}$are defined in the laboratory rest frame with respect to the particle's velocity. $G=\frac{g-2}{2}$ is the anomalous magnetic moment coefficient, and $\gamma m c^{2}$ is the energy of the particle. Neglecting the electric field, we can transform this equation by expanding about a reference orbit described by the Frenet-Serret coordinate system shown in Fig. (1). Thus we have

$$
\frac{d \hat{x}}{d s}=\frac{\hat{s}}{\rho}, \quad \frac{d \hat{s}}{d s}=-\frac{\hat{x}}{\rho}, \text { and } \frac{d \hat{z}}{d s}=0
$$

where $\rho$ is the local radius of curvature for the reference orbit. Particle motion can be parameterized in this coordinate system as,

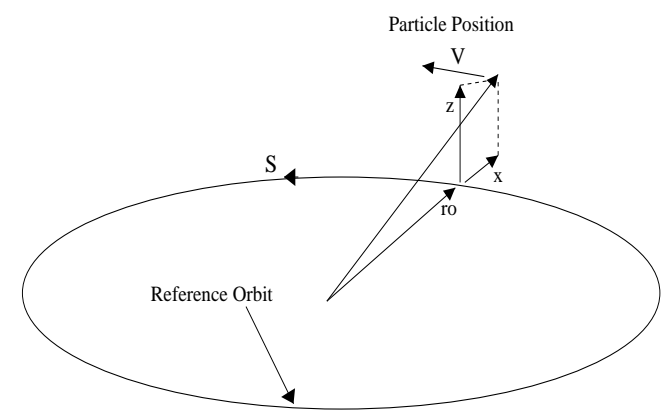

FIG. 1: The curvilinear coordinate system for a particle motion in a circular accelerator. $\hat{x}, \hat{s}$ and $\hat{z}$ are the transverse radial, the longitudinal, and the transverse vertical unit base vectors, and $\vec{r}_{0}(s)$ is the reference orbit. 


$$
\vec{r}=\vec{r}_{o}(s)+x \hat{x}+z \hat{z}
$$

Here $\vec{r}_{o}(s)$ is the reference orbit and $\hat{s}=d \vec{r}_{o} / d s$. The velocity becomes

$$
\begin{aligned}
\vec{v} & =\frac{d \vec{r}}{d t} \\
& =\frac{d s}{d t}\left(x^{\prime} \hat{x}+\left(1+\frac{x}{\rho}\right) \hat{s}+z^{\prime} \hat{z}\right) \\
& \approx v\left(x^{\prime} \hat{x}+\hat{s}+z^{\prime} \hat{z}\right) \\
\vec{v}^{\prime} & =v\left(\left(x^{\prime \prime}-\frac{1}{\rho}\right) \hat{x}+\frac{x^{\prime}}{\rho} \hat{s}+z^{\prime \prime} \hat{z}\right) .
\end{aligned}
$$

All primes ' represent derivatives with respect to $s$. The transverse magnetic field can now be expressed as,

$$
\begin{aligned}
\vec{B}_{\perp} & =\frac{1}{v^{2}}(\vec{v} \times \vec{B}) \times \vec{v} \\
& =B \rho\left(1-\frac{x}{\rho}\right)\left[\left(x^{\prime \prime}-\frac{1}{\rho}\right) \hat{z}+\frac{z^{\prime}}{\rho} \hat{s}-z^{\prime \prime} \hat{x}\right]
\end{aligned}
$$

Where we have made use of $\frac{d s}{d t} \approx v(1-x / \rho)$ and $\vec{v} \times \vec{B}=$ $\frac{\gamma m c}{e} \frac{d \vec{v}}{d t}$. We should also note that $B_{\perp} \rho=\gamma m c v / e$ is the magnetic rigidity of the particle. To first order, $\vec{B}_{\|}$can be found to be,

$$
\vec{B}_{\|} \approx\left(B_{s}+B_{z} z^{\prime}\right) \hat{s}
$$

Using the dipole guiding field $B_{z}=-B_{\perp} \rho / \rho$, the $B_{s}$ field can be derived from Maxwell's equations obtaining,

$$
\begin{gathered}
\frac{\partial B_{s}}{\partial z}=\frac{\partial B_{z}}{\partial s}=-(B \rho)\left(\frac{1}{\rho}\right)^{\prime} \\
B_{s}=-B \rho z\left(\frac{1}{\rho}\right)^{\prime} .
\end{gathered}
$$

Neglecting higher order terms,

$$
\vec{B}_{\|} \approx-B \rho\left(\frac{z}{\rho}\right)^{\prime} \hat{s}
$$

Then using $\frac{d}{d t}=\frac{v}{\rho+x} \frac{d}{d \theta}$ the Thomas-BMT equation becomes

$$
\frac{d \vec{S}}{d \theta}=\vec{S} \times \vec{F}
$$

where $\vec{F}=F_{1} \hat{x}+F_{2} \hat{s}+F_{3} \hat{z}$ and the elements are

$$
\begin{aligned}
& F_{1}=-\rho z^{\prime \prime}(1+G \gamma) \\
& F_{2}=(1+G \gamma) z^{\prime}-\rho(1+G)\left(\frac{z}{\rho}\right)^{\prime} \\
& F_{3}=-(1+G \gamma)+(1+G \gamma) \rho x^{\prime \prime} .
\end{aligned}
$$

Using $\frac{d \hat{x}}{d \theta}=\hat{s}$, and $\frac{d \hat{s}}{d \theta}=-\hat{x}$ Eq. (12) becomes

$$
\begin{aligned}
& \frac{d S_{1}}{d \theta}=\left(1+F_{3}\right) S_{2}-F_{2} S_{3}, \\
& \frac{d S_{2}}{d \theta}=-\left(1+F_{3}\right) S_{1}+F_{1} S_{3}, \\
& \frac{d S_{3}}{d \theta}=F_{2} S_{1}-F_{1} S_{2} .
\end{aligned}
$$

Expressed in the rotating frame Eq (12) then becomes

$$
\frac{d \vec{S}}{d \theta}=\vec{n} \times \vec{S}
$$

Where $\vec{n}=-\left[F_{1} \hat{x}+F_{2} \hat{s}+\left(1+F_{3}\right) \hat{z}\right]$. Since we are concerned only with spin $1 / 2$ particles we can employ the well developed spinor formalism. Using the Pauli matrices

$$
\sigma_{x}=\left(\begin{array}{cc}
0 & 1 \\
1 & 0
\end{array}\right), \sigma_{s}=\left(\begin{array}{cc}
0 & -i \\
i & 0
\end{array}\right), \text { and } \sigma_{z}=\left(\begin{array}{cc}
1 & 0 \\
0 & -1
\end{array}\right)
$$

the polarization can be given by

$$
\vec{S}=\Psi^{\dagger} \vec{\sigma} \Psi
$$

Substituting Eq. (17) into the left side of Eq. (15) yields

$$
\frac{d \vec{S}}{d \theta}=\frac{d \Psi^{\dagger}}{d \theta} \vec{\sigma} \Psi+\Psi^{\dagger} \vec{\sigma} \frac{d \Psi}{d \theta} .
$$

Using $[\vec{\sigma} \cdot \vec{n}, \vec{\sigma}]=2 i(\vec{n} \times \sigma)$, the right hand side becomes

$$
\vec{n} \times \vec{S}=-\frac{i}{2}\left(\Psi^{\dagger} \vec{\sigma}\right) \vec{\sigma} \cdot \vec{n} \Psi+\frac{i}{2} \Psi^{\dagger} \vec{\sigma} \cdot n(\vec{\sigma} \Psi) .
$$

Finally equating both sides gives

$$
\frac{d \Psi}{d \theta}=-\frac{i}{2}(\vec{\sigma} \cdot \vec{n}) \Psi=-\frac{i}{2}\left(\begin{array}{cc}
f_{3} & -\xi \\
\xi^{*} & -f_{3}
\end{array}\right) \Psi
$$

Where $\xi(\theta)=F_{1}-i F_{2}$ and $f_{3}=\left(1+F_{3}\right)$. Although the spinor wave function $\Psi$ is similar in form to the quantum mechanical state function, in this case $\vec{S}$ is a classical vector. As in the quantum mechanical case, however, this two component spinor is defined,

$$
\Psi=\left(\begin{array}{l}
u \\
d
\end{array}\right) \text {. }
$$

Here $u$ and $d$ are complex numbers representing the up and down components. The components of the spin vector become

$$
\begin{aligned}
& S_{1}=u^{*} d+u d^{*} \\
& S_{2}=-i\left(u^{*} d-u d^{*}\right) \\
& S_{3}=|u|^{2}-|d|^{2} .
\end{aligned}
$$

Because $H=(\vec{\sigma} \cdot \vec{n})$ is hermitian,

$$
|\vec{S}|=|u|^{2}+|d|^{2}=\Psi^{\dagger} \Psi
$$

and the magnitude of the spin vector remains constant. The normalization condition for the spinor wave function is chosen to be $\Psi^{\dagger} \Psi=1$. 


\section{RECASTING TO LINEAR 2ND ORDER DIFFERENTIAL EQUATION}

Moving to the Interaction frame using the following transformation:

$$
\begin{array}{r}
\Psi(\theta)=e^{-\frac{i}{2} \int_{0}^{\theta} f_{3}(t) d t \hat{\sigma}_{z}} \Psi_{I}(\theta) \\
\hat{\xi}(\theta)=\xi(\theta) e^{i \int_{0}^{\theta} f_{3}(t) d t \hat{\sigma}_{z}}
\end{array}
$$

This yields two first order coupled differential equations:

$$
\begin{aligned}
\frac{d \Psi_{I}^{+}}{d \theta} & =\frac{i}{2} \hat{\xi} \Psi_{I}^{-} \\
\frac{d \Psi_{I}^{-}}{d \theta} & =\frac{i}{2} \hat{\xi}^{*} \Psi_{I}^{+}
\end{aligned}
$$

These equations can be cast into a standard 2nd order homogeneous linear Differential equation with variable coefficients.

$$
\frac{d^{2} \Psi_{I}^{+}}{d \theta^{2}}-\left(i f_{3}(\theta)+\frac{\xi^{\prime}(\theta)}{\xi(\theta)}\right) \frac{d \Psi_{I}^{+}}{d \theta}+\frac{\xi(\theta) \xi(\theta)^{*}}{4} \Psi_{I}^{+}=0(26)
$$

\section{TRANSFORMATION TO HILLS' LIKE DIFFERENTIAL EQUATION}

It is possible to eliminate the 1st order derivative in Eq. (26) to obtain a Hill's like differential equation, using the following definition:

$$
\begin{aligned}
\beta(\theta) & =-\left(i f_{3}(\theta)+\frac{\xi^{\prime}(\theta)}{\xi(\theta)}\right) \\
\Omega^{2}(\theta) & =\frac{\beta^{\prime}(\theta)}{2}+\frac{\beta(\theta)^{2}}{4}-\frac{\xi(\theta) \xi(\theta)^{*}}{4} \\
D(\theta) & =\frac{1}{2} \int^{t} d \tau \beta(\tau) \\
q(\theta) & =e^{D(\theta)} \Psi_{I}^{+}(\theta) \\
\frac{d^{2} q}{d \theta^{2}} & =\Omega^{2}(\theta) q
\end{aligned}
$$

Evaluating $\Omega^{2}(\theta)$ we get:

$$
\begin{aligned}
& \Omega^{2}(\theta)=-\frac{i f_{3}^{\prime}}{2}-\frac{f_{3}^{2}}{4}+i \frac{f_{3}}{2}\left(\frac{\xi^{\prime}(\theta)}{\xi(\theta)}\right)+ \\
& \frac{3}{4}\left(\frac{\xi^{\prime}(\theta)}{\xi(\theta)}\right)^{2}-\frac{1}{2}\left(\frac{\xi^{\prime \prime}(\theta)}{\xi(\theta)}\right)-\frac{\xi(\theta) \xi(\theta)^{*}}{4}
\end{aligned}
$$

Considering the form of Eq. (28) we can see that $\Omega^{2}(\theta)$ will have oscillating terms from the transverse motion due to $z$. In fact the standard approach is to expand $F_{1}-i F_{2}$ into a fourier series:

$$
\xi(\theta)=F_{1}-i F_{2}=\sum_{K} \varepsilon_{K} e^{-i K \theta}
$$

where the Fourier coefficient or resonance strength $\varepsilon_{K}$ is given by,

$\varepsilon_{K}=-\frac{1}{2 \pi} \oint\left[(1+G \gamma)\left(\rho z^{\prime \prime}+i z^{\prime}\right)-i \rho(1+G)\left(\frac{z}{\rho}\right)^{\prime}\right] e^{i K \theta} d \theta$

and $K$ is the resonance spin tune. Also usually to first order the $(1+G \gamma) \rho x^{\prime \prime}$ term is ignored. In the case when $G \gamma$ is constant the equation reduces to a normal Hills equation with a constant piece defined by $G \gamma$ and the oscillating piece given by the Fourier expansion. In this case we have parametric resonances when ever the constant or polynomial $f_{3}$ terms equal the frequency of the $\xi$ terms. For a single resonance the oscillating pieces coming from $\frac{\xi^{\prime}(\theta)}{\xi(\theta)}$ and $\frac{\xi(\theta) \xi(\theta)^{*}}{4}$ cancel and the whole of $\Omega(\theta)$ is constant and becomes a simple harmonic oscillator. In the case $G \gamma(\theta)$ is not constant but we still have only a single resonance term in $\xi(\theta)$ then $\Omega^{2}(\theta)$ again all oscillating terms cancel as before but we acquire a polynomial in $\theta$. If the acceleration is linear then it is a 2nd order polynomial. This of course could be solved either using Eq. (26) (the standard Froissart-Stora method) with hypergeometic solutions or in the current form Eq. (27) solutions are Parabolic Cylinder Functions. In the case when you have both accelerating $G \gamma$ and multiple frequency terms in $\xi$ then $\Omega^{2}(\theta)$ has both polynomial and oscillating terms which make the solution much more challenging.

However following [4] who considered a similar system with linear terms and multiple frequency terms, we can break up $\Omega^{2}$ into regions where the polynomial terms dominate and where the parametric resonance dominates and attempt a piece wise approximation which captures the important dynamics of this system.

\section{A. Approximation for $\Omega^{2}$}

Since the $\frac{1}{\xi(\theta)}$ term presents most problem, we will expand the denominator to first order to obtain a adequate approximation for this term. Normally in the RHIC lattice we deal with the overlap of a strong intrinsic resonance with a smaller nearby intrinsic or imperfection resonance. Considering for now only two resonances one strong and the other weak we expand to first order in the small parameter $\epsilon=a_{2} / a_{1}$ where $a_{1}$ is the absolute value of the stronger resonances $\varepsilon_{K_{1}}$ and $a_{2}$ the weaker.

$$
\begin{aligned}
\frac{1}{\xi(\theta)} & =\frac{1}{a_{1} e^{-i\left(K_{1} \theta+\phi_{1}\right)}+a_{2} e^{-i\left(K_{2} \theta+\phi_{2}\right)}} \\
& \approx \frac{e^{i\left(K_{1} \theta+\phi_{1}\right)}}{a_{1}}\left(1-\left(\epsilon e^{i \Delta \phi}\right) e^{i \delta \theta}\right)
\end{aligned}
$$

Here $\phi_{1,2}$ are the phases of the $\varepsilon_{K_{1}, K_{2}}, \Delta \phi=\phi_{1}-\phi_{2}$ and $\delta=K_{1}-K_{2}$ and expanded to first order in $\epsilon$. Now we can generate a new approximate $\beta(\theta)$,

$$
\beta(\theta) \approx-\left(i f_{3}(\theta)-i K_{1}+i \delta \epsilon e^{i \delta \theta}\right)
$$


Keeping only first order terms in $\epsilon$ our approximate $\Omega(\theta)^{2}$ becomes,

$$
\begin{aligned}
\Omega^{2}(\theta) & \approx W_{0}^{2}+C_{1} \theta+C_{2} \theta^{2}+C_{3} e^{i \delta \theta}+C_{4} \theta e^{i \delta \theta}+C_{5} e^{-i \delta \theta} \\
W_{0}^{2} & =-i \frac{\alpha}{2}-\frac{\kappa_{0}^{2}}{4}+\frac{K_{1} \kappa_{0}}{2}-\frac{K_{1}^{2}}{4}-\frac{a_{1}^{2}}{4}-\frac{a_{2}^{2}}{4} \\
C_{1} & =\alpha \frac{K_{1}-\kappa_{0}}{2} \\
C_{2} & =-\frac{\alpha^{2}}{4} \\
C_{3} & =\left(-\frac{a_{1}^{2}}{4}+\frac{\delta^{2}}{2}+\frac{\delta\left(K_{1}-\kappa_{0}\right)}{2}\right) \epsilon_{p} \\
C_{4} & =-\frac{\alpha \delta}{2} \epsilon_{p} \\
C_{5} & =-\frac{a_{1}^{2}}{4} \epsilon_{m} \\
\epsilon_{p} & =\epsilon e^{i \Delta \phi} \\
\epsilon_{m} & =\epsilon e^{-i \Delta \phi}
\end{aligned}
$$

Here we have developed a general $\Omega^{2}$ considering when $G \gamma$ is ramping constantly and we can define $f_{3}(\theta)=\kappa_{0}+$ $\alpha \theta$. With the initial $G \gamma_{0}=\kappa_{0}$

\section{DERIVATION OF APPROXIMATE SOLUTION NON ACCELERATING CASE}

In case that $G \gamma$ is constant $(\alpha=0), C_{1}=C_{2}=C_{4}=$ 0 and $\Omega^{2}$ reduces to,

$$
\Omega^{2}(\theta) \approx W_{0}^{2}+C_{3} e^{i \delta \theta}+C_{5} e^{-i \delta \theta}
$$

In this case the second to terms become important when $W_{0}^{2}=\delta^{2} / 4$ in which case you have a $2: 1$ parametric resonance out side of this region we have a normal harmonic oscillator with frequency $W_{0}$. If $C_{5} \approx 0$ or $C_{3} \approx 0$ then solutions are Mathieu functions. Otherwise a solution can be developed using Hills approach by expanding $q$ in a Fourier series and matching terms. However a decent approximation is possible using a multi scale method if we assume $C_{3}>C_{5}$ and $C_{3}<1$. In this case we can introduce two new time parameters, $\eta=C_{3} \theta$ and $\theta=t$ we also introduce $\epsilon_{0}=C_{5} / C_{3}$ to obtain (in some cases $C_{5}>C_{3}$, in which case $C_{5}$ is factored out and $\left.\epsilon_{0}=C_{3} / C_{5}\right)$,

$$
\frac{\partial^{2} q}{\partial t^{2}}+2 C_{3} \frac{\partial^{2} q}{\partial t \partial \eta}-\left(W_{0}^{2}+C_{3}\left(e^{i \delta t}+\epsilon_{0} e^{-i \delta t}\right)\right) q=0
$$

Expanding $q=q_{0}+C_{3} q_{1}$ and collecting $C_{3}$ terms of the same order we get,

$$
\begin{aligned}
& \frac{\partial^{2} q_{0}}{\partial t^{2}}-W_{0}^{2} q_{0}=0 \\
& \frac{\partial^{2} q_{1}}{\partial t^{2}}-W_{0}^{2} q_{1}=-2 \frac{\partial^{2} q_{0}}{\partial t \partial \eta}+\left(e^{i \delta t}+\epsilon_{0} e^{-i \delta t}\right) q_{0}
\end{aligned}
$$

near the parametric resonance $W_{0}^{2} \approx-\delta^{2} / 4$ in which case,

$$
q_{0}(t)=A(\eta) e^{i \delta t / 2}+B(\eta) e^{-i \delta t / 2}
$$

Canceling the secular terms in the $q_{1}$ equation leads to a pair of coupled first order equations for $A$ and $B$ coefficients:

$$
\begin{aligned}
i \delta \frac{d A}{d \eta} & =-B \\
i \delta \frac{d B}{d \eta} & =\epsilon_{0} A
\end{aligned}
$$

the solutions are,

$$
\begin{aligned}
& A=e^{\sqrt{\epsilon_{0}} \eta / \delta} \\
& B=-i \sqrt{\epsilon_{0}} e^{\sqrt{\epsilon_{0}} \eta / \delta}
\end{aligned}
$$

So an approximation for $q$ when we are at parametric resonance becomes,

$$
\begin{array}{r}
q(\theta)=A_{1} e^{\sqrt{\epsilon_{0}} C_{3} \theta / \delta}\left(e^{i \delta \theta / 2}-i \sqrt{\epsilon_{0}} e^{-i \delta \theta / 2}\right)+ \\
A_{2} e^{-\sqrt{\epsilon_{0}} C_{3} \theta / \delta}\left(e^{i \delta \theta / 2}+i \sqrt{\epsilon_{0}} e^{-i \delta \theta / 2}\right)
\end{array}
$$

Here $A_{1,2}$ are determined by the boundary conditions. For the case when $C_{5}>C_{3}$ it becomes:

$$
\begin{array}{r}
q(\theta)=A_{1} e^{\sqrt{\epsilon_{0}} C_{5} \theta / \delta}\left(e^{-i \delta \theta / 2}-i \sqrt{\epsilon_{0}} e^{i \delta \theta / 2}\right)+ \\
A_{2} e^{-\sqrt{\epsilon_{0}} C_{5} \theta / \delta}\left(e^{-i \delta \theta / 2}+i \sqrt{\epsilon_{0}} e^{i \delta \theta / 2}\right)
\end{array}
$$

and $\epsilon_{0}=C_{3} / C_{5}$. This approximation becomes valid when we are in the neighborhood of a resonance tongue,

$$
W_{0}^{2} \approx \delta^{2} / 4 \pm\left|C_{a}\right| / 2
$$

Where $C_{a}=C_{3}$ is $C_{3}>C_{5}$ or $C_{a}=C_{5}$ if $C_{3}<C_{5}$. Outside of this region we can approximate with a simple Harmonic oscillator of frequency $W_{0}^{2}$.

\section{DERIVATION OF APPROXIMATE SOLUTION FOR ACCELERATING CASE}

In this case as with the previous case we see that outside of the parametric resonant tongue region we can ignore the oscillatory pieces of $\Omega^{2}$. The approximate $\Omega^{2}$ for this region becomes,

$$
\Omega_{c}^{2}(\theta) \approx W_{0}^{2}+C_{1} \theta+C_{2} \theta^{2}
$$

For this $\Omega_{c}^{2}$ solutions exist in the form of so called Parabolic Cylinder functions $D_{\mu}(x)$. The solution is:

$$
\begin{aligned}
q_{c}(\theta) & \approx A_{1} D_{\mu_{-}}(y(\theta))+A_{2} D_{\mu_{+}}(i y(\theta)) \\
\mu_{ \pm} & =\frac{ \pm\left(C_{1}^{2}-4 C_{2} W_{0}^{2}\right)-4 C_{2}^{3 / 2}}{8 C_{2}^{3 / 2}} \\
y(\theta) & =\frac{C_{1}}{\sqrt{2} C_{2}^{3 / 4}}+\sqrt{2} C_{2}^{1 / 4} \theta
\end{aligned}
$$


with $A_{1,2}$ constants due to boundary conditions. For this case the location of the parametric resonance is $\theta=\theta_{r}$

$$
\theta_{r \pm}=\frac{-C_{1} \pm \sqrt{C_{1}^{2}-C_{2} \delta^{2}-4 C_{2} W_{0}^{2}}}{2 C_{2}}
$$

If both $\theta_{r \pm}$ roots are positive then there are two parametric resonances and the region around each tongue is defined as $\theta_{1 \pm}$ and $\theta_{2 \pm}$,

$$
\begin{aligned}
& \theta_{1 \pm} \approx \frac{-C_{1} \pm \sqrt{C_{1}^{2}-2 C_{2}\left|C_{a}\right|-C_{2} \delta^{2}-4 C_{2} W_{0}^{2}}}{2 C_{2}} \\
& \theta_{2 \pm} \approx \frac{-C_{1} \pm \sqrt{C_{1}^{2}+2 C_{2}\left|C_{a}\right|-C_{2} \delta^{2}-4 C_{2} W_{0}^{2}}}{2 C_{2}}(46)
\end{aligned}
$$

Defining $C_{r}=C_{3}+C_{4} \theta_{r}, C_{a}=C_{r}$ if $\left|C_{r}\right|>\left|C_{5}\right|$ else $C_{a}=C_{5}$. So for the case when $\left|C_{r}\right|>\left|C_{5}\right|$ Eq. becomes,

$$
\begin{array}{r}
q(\theta)=A_{1} e^{\sqrt{\epsilon_{0}} C_{r} \theta / \delta}\left(e^{i \delta \theta / 2}-i \sqrt{\epsilon_{0}} e^{-i \delta \theta / 2}\right)+ \\
A_{2} e^{-\sqrt{\epsilon_{0}} C_{r} \theta / \delta}\left(e^{i \delta \theta / 2}+i \sqrt{\epsilon_{0}} e^{-i \delta \theta / 2}\right)
\end{array}
$$

with $\epsilon_{0}=C_{5} / C_{r}$ and when $\left|C_{r}\right|<\left|C_{5}\right|$ we recover Eq. 41 with now $\epsilon_{0}=C_{r} / C_{5}$.

\section{CONSTRUCTING A FULL SOLUTION}

Matching the boundary conditions for $\Psi_{I}^{+}\left(\theta_{0}\right)=B_{c 1}$ and $\Psi_{I}^{+\prime}\left(\theta_{0}\right)=B_{c 2}$ becomes $q\left(\theta_{0}\right)=e^{D\left(\theta_{0}\right)} B_{c 1}$ and $q^{\prime}\left(\theta_{0}\right)=\frac{\beta\left(\theta_{0}\right) e^{D\left(\theta_{0}\right)}}{2} B_{c 2}$. Generally the constants for a solution to a second order differential equation can be determined from the inverted Wronskian $\left(W^{-1}(\theta)\right)$ at the $\theta=\theta_{0}$ location of the boundary conditions.

$$
\begin{aligned}
\left(\begin{array}{c}
q\left(\theta_{0}\right) \\
q^{\prime}\left(\theta_{0}\right)
\end{array}\right) & =\left(\begin{array}{ll}
q_{1}\left(\theta_{0}\right) & q_{2}\left(\theta_{0}\right) \\
q_{1}^{\prime}\left(\theta_{0}\right) & q_{2}^{\prime}\left(\theta_{0}\right)
\end{array}\right)\left(\begin{array}{c}
B_{c 1} \\
B_{c 2}
\end{array}\right) \\
\vec{Q}\left(\theta_{0}\right) & =\vec{W}\left(\theta_{0}\right) \vec{B}_{c} \\
\vec{W}^{-1}\left(\theta_{0}\right) \vec{Q}\left(\theta_{0}\right) & =\vec{B}_{c}
\end{aligned}
$$

Here $q_{1,2}$ are the particular solutions and $B_{c 1,2}$ the boundary conditions specific for $q\left(\theta_{0}\right), q^{\prime}\left(\theta_{0}\right)$. Matching the boundaries between each region we can construct a piecewise solution using Eq. $(44,47,41)$. We split up the solution into three regions Region I: $0<\theta<\theta_{-}$, Region II: $\theta_{-}<\theta<\theta_{+}$and Region III: $\theta>\theta_{+}$.

Beginning in Region I we use the Parabolic Cylinder solution of Eq. (44) and match to the initial boundary conditions at $\Psi^{+}(0)=\Psi_{I}^{+}(0)=1$ and $\Psi^{+\prime}(0)=$ $\Psi_{I}^{+\prime}(0)=0$. This becomes $q(0)=e^{D(0)}$ and $q^{\prime}(0)=0$. Then using the Wronskian approach shown in Eq. (48), the constants $\left(A_{1}, A_{2}\right)$ become $\vec{A}=\vec{W}_{c}^{-1}(0) \vec{Q}(0)$. Here $W_{c}(\theta)$ is made up of the two Parabolic Cylinder particular solutions shown in Eq. (44).

In Region II we use the Hills' like approximation equations of Eq. (47) or (41) depending on whether the negative or positive frequency amplitude is larger. Here we have to match Region I equations at the boundary $\theta_{-}$. So the constants in this region become $\vec{A}=$ $\vec{W}_{p}^{-1}\left(\theta_{-}\right) \vec{Q}_{c}\left(\theta_{-}\right)$. Here $\vec{W}_{p}(\theta)$ is the Wronskian made up from particular solutions given in Eq. (47) or (41) and $\vec{Q}_{c}$ denotes the solutions in region I.

Finally in region III the solution goes back to the Parabolic Cyclinder form matching from region II at $\theta_{+}$ to get the constants $\vec{A}=\vec{W}_{c}^{-1}\left(\theta_{+}\right) \vec{Q}_{p}\left(\theta_{+}\right)$.

\section{COMPARISON OF THE APPROXIMATE SOLUTION AGAINST NUMERICALLY INTEGRATED SOLUTIONS}

For the RHIC machine there are several particular manners in which two resonances may interfere. We consider cases where the resonances are less than or equal to one unit of $G \gamma$ away. This would include neighboring intrinsic resonances or imperfection resonances. We can classify cases based on the location of the parametric resonances relative to the primary spin resonance and the absolute strength of the primary resonance which can effect the threshold and overall effect of the parametric resonances. In Fig. (2)-(4) we can see the approximation and direct numerical solution for the case when the primary resonance is more than strong enough for a spin flip and it see's a second single parametric resonance.

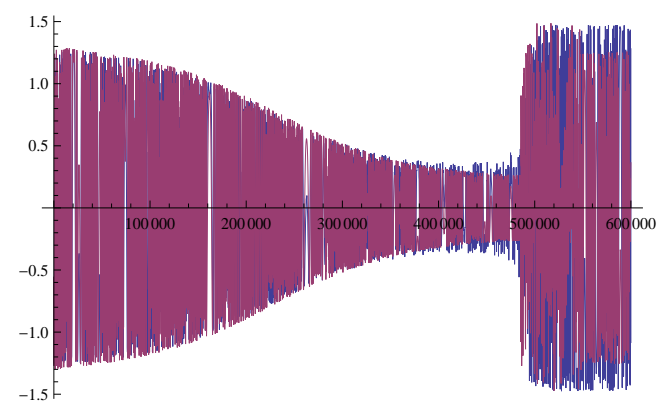

FIG. 2: Plot of $\operatorname{Im}(\mathrm{q})$ versus $\theta$ with $\kappa_{0}=414.0, K_{1}=414.673$ and $K_{2}=415.673, a_{1}=0.45, a_{2}=0.01$ and $\Delta \phi=0$ and $\alpha=3.18 \times 10^{-6}$. Blue trace is direct numerical integration, Purple trace piece wise approximation

In Fig. (5) we show the case when there is no parametric resonance since $\Omega_{c}^{2}$ never equals $\frac{\delta^{2}}{4}$. In this case the Parabolic cylindrical Solution works fine by itself. This only breaks down when the size of the $a_{2}$ becomes large enough for the truncated $\epsilon^{2}$ terms to contribute and bring in higher frequency terms. We can then see the onset of parametric resonances when $\Omega_{c}^{2}=\delta^{2}$.

In Fig. (6) we show the case when both roots are greater than $\kappa_{0}$ our starting $G \gamma$ value, for $\theta_{r \pm}$. In this case the first parametric resonance occurs before the onset of the primary resonances followed by a second parametric resonance. 


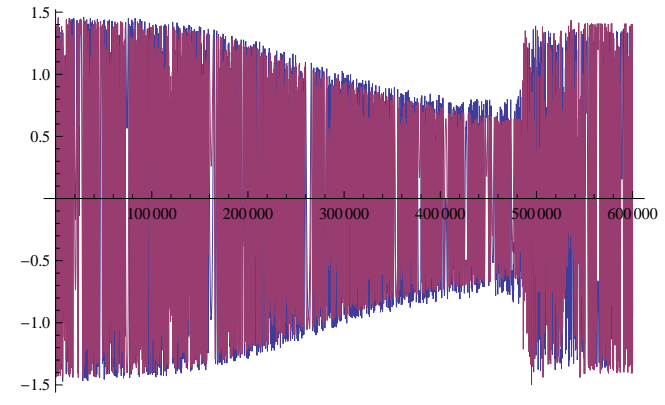

FIG. 3: Plot of $\operatorname{Re}(\mathrm{q})$ versus $\theta$ with $\kappa_{0}=414.0, K_{1}=414.673$ and $K_{2}=415.673, a_{1}=0.45, a_{2}=0.01$ and $\Delta \phi=0$ and $\alpha=3.18 \times 10^{-6}$. Blue trace is direct numerical integration, Purple trace piece wise approximation

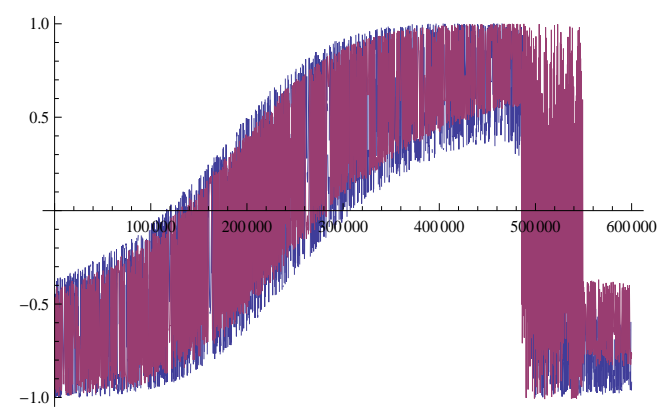

FIG. 4: $S_{y}$ versus $\theta$ with $\kappa_{0}=414.0, K_{1}=414.673$ and $K_{2}=415.673, a_{1}=0.45, a_{2}=0.01$ and $\Delta \phi=0$ and $\alpha=$ $3.18 \times 10^{-6}$. Blue trace is direct numerical integration, Purple trace piece wise approximation

\section{INTEGRATING OVER DISTRIBUTION OF PARTICLES WITH DIFFERENT INITIAL BETATRON PHASES}

This Hills form for the differential equation also has the added benefit that it is fairly easy to integrate out the effect of a distribution of particles with different initial betatron phases given by $\phi_{1}$ or $\phi_{2}$ since inside the differential equation for $q$ the kernel is function of only

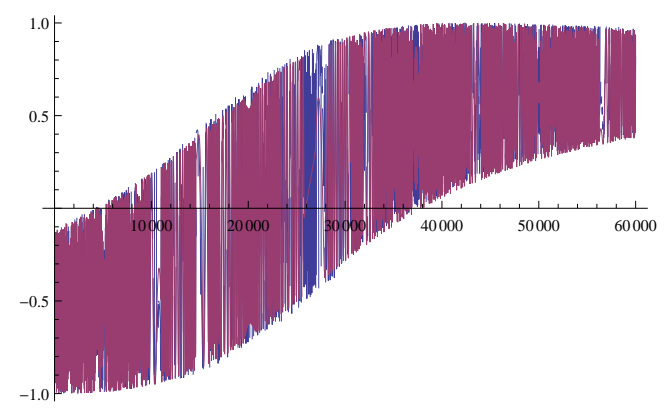

FIG. 5: $S_{y}$ versus $\theta$ with $\kappa_{0}=414.0, K_{1}=414.673$ and $K_{2}=$ 415.0, $a_{1}=0.6, a_{2}=0.01$ and $\Delta \phi=0$ and $\alpha=3.18 \times 10^{-5}$. Blue trace is direct numerical integration, Purple trace piece wise approximation

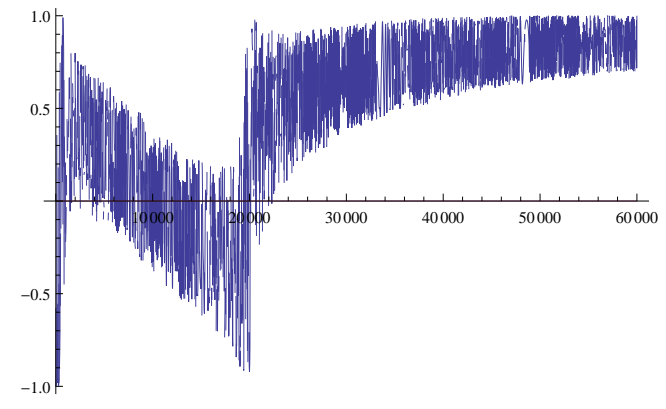

FIG. 6: Showing the case with a second non-negative root causing two parametric resonances: One before normal resonance the other after. $S_{y}$ versus $\theta$ with $\kappa_{0}=414.0$, $K_{1}=414.327$ and $K_{2}=415.0, a_{1}=0.6, a_{2}=0.01$ and $\Delta \phi=\pi / 4$ and $\alpha=3.18 \times 10^{-5}$. Blue trace is direct numerical integration (no approximation is shown).

the difference $\Delta \phi$ and the individual phases are in $D(\theta)$ only. So we can integrate out $\phi_{2}$ as follows,

$$
\begin{aligned}
& \int \Psi_{I}^{+} d \phi_{2}=\int e^{D\left(\theta, \phi_{2}\right)} q(\theta) d \phi_{2} \\
& \int \Psi_{I}^{+} d \phi_{2}=q(\theta) \int e^{D\left(\theta, \phi_{2}\right)} d \phi_{2}
\end{aligned}
$$

For the case of two resonances this can be performed analytically with $D(\theta)$ becoming:

$$
\begin{aligned}
D\left(\theta, \phi_{2}\right)= & \frac{1}{2} \int^{\theta} d \tau \beta(\tau) \\
D\left(\theta, \phi_{2}\right)= & -\frac{i}{2}\left(\frac{1}{2} \theta\left(2 \kappa_{0}-2 K_{1}-2 K_{2}+\alpha \theta\right)-\right. \\
& \left.i \ln \left(a_{2} e^{i\left(\Delta \phi+\phi_{2}+K_{1} \theta\right)}+a_{1} e^{i\left(\phi_{2}+K_{2} \theta\right.}\right)\right)(50)
\end{aligned}
$$

and then Integrating,

$$
\int e^{D\left(\theta, \phi_{2}\right)} d \phi_{2}=\frac{2 i e^{-\frac{i}{4} \theta\left(2 \kappa_{0}-2 K_{1}-2 K_{2}+\alpha \theta\right)}}{\sqrt{a_{2} e^{i\left(\Delta \phi+\phi_{2}+K_{1} \theta\right)}+a_{1} e^{i\left(\phi_{2}+K_{2} \theta\right)}}}(51)
$$

\section{ISSUES MODELING PARAMETRIC RESONANCE CROSSINGS}

While this approach can identify the location and relative strength of the parametric resonances. Modeling the actual crossing is still problematic. This is because the current approximation doesn't capture the gradual blow up in $q$ amplitude during the acceleration across the resonance and the phase can often be incorrect when it is matched to the out-going parabolic cylinder solution. This can result in under or over-estimating the blow up (See Fig. (7) - (8)

More work still needs to be done to develop a reliable estimate for the blowup. We can either approach this problem empirically and develop response by scanning through different cases and phases or use approximations 


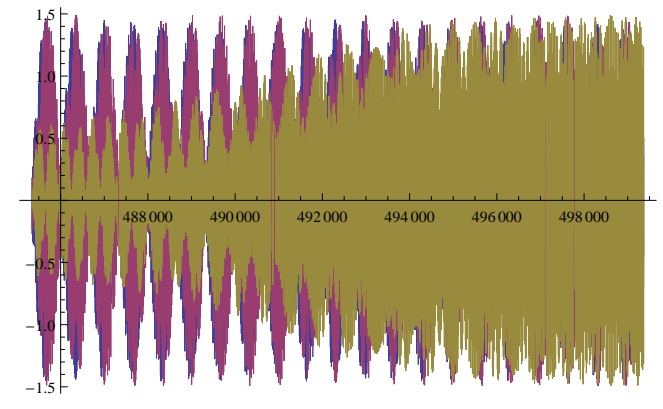

FIG. 7: Parametric Resonances crossing: Plot of $\operatorname{Im}(\mathrm{q})$ versus $\theta$ with $\kappa_{0}=414.0, K_{1}=414.673$ and $K_{2}=415.673$, $a_{1}=0.45, a_{2}=0.01$ and $\Delta \phi=0$ and $\alpha=3.18 \times 10^{-6}$. Yellow trace is direct numerical integration, Purple trace stationary Parametric approximation, blue numerical stationary parametric solution

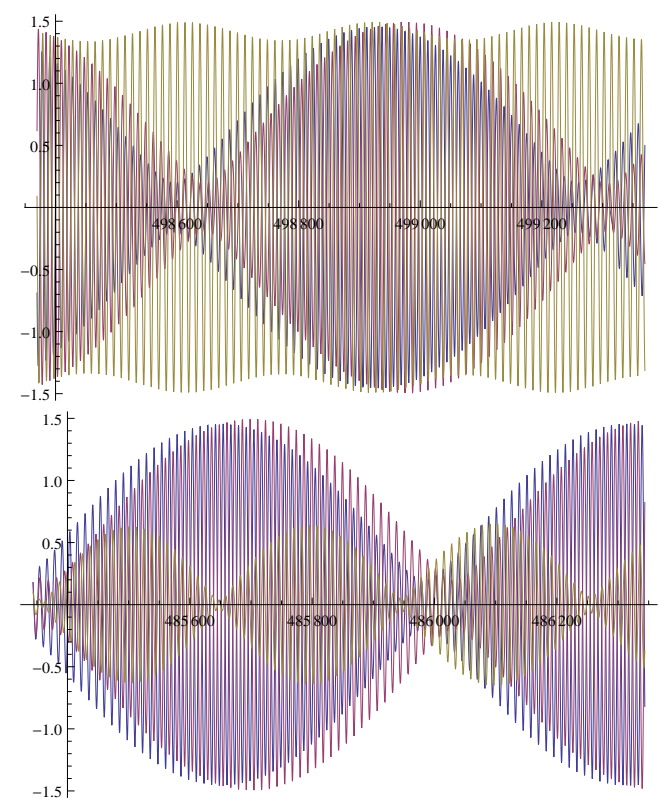

FIG. 8: Zoom of end (top) and begining (bottom) of Parametric Resonances crossing: Plot of $\operatorname{Im}(\mathrm{q})$ versus $\theta$ with $\kappa_{0}=414.0, K_{1}=414.673$ and $K_{2}=415.673, a_{1}=0.45$ $a_{2}=0.01$ and $\Delta \phi=0$ and $\alpha=3.18 \times 10^{-6}$. Yellow trace is direct numerical integration, Purple trace stationary Parametric approximation, blue numerical stationary parametric solution

developed for FFAG parametric resonance crossings [5] and recast Hills' form into a Hamiltonian form and then go to action angles.

\section{ESTIMATE OF BLOW-UP USING ACTION ANGLE}

We can cast Eq. (27) in a Hamiltonian formalism:

If we define:

$$
H=\frac{q^{\prime 2}}{2}-\frac{\Omega^{2}(\theta) q^{2}}{2}
$$

$$
Q^{2}(\theta)=-W_{0}^{2}-C_{1} \theta-C_{2} \theta^{2}
$$

The Hamiltonian becomes:

$H=\frac{q^{\prime 2}}{2}+\frac{Q^{2}(\theta) q^{2}}{2}-\frac{q^{2}}{2}\left(C_{3} e^{i \delta \theta}+C_{4} \theta e^{i \delta \theta}+C_{5} e^{-i \delta \theta}(54)\right.$

Now casting this in terms of action angles $(J$ and $\Psi)$ :

$$
\begin{aligned}
q & =\sqrt{2 J / Q} \cos \Psi \\
q^{\prime} & =\sqrt{2 J Q} \sin \Psi \\
H & =Q J-\frac{J}{Q} \cos ^{2} \Psi\left(C_{3} e^{i \delta \theta}+C_{4} \theta e^{i \delta \theta}+C_{5} e^{-i \delta \theta}(55)\right.
\end{aligned}
$$

Then finding equations of motion:

$$
\begin{aligned}
\Psi^{\prime}= & \frac{\partial H}{\partial J} \approx Q(\theta) \\
\Psi(\theta) \approx & \int \sqrt{-W_{0}^{2}-C_{1} \theta-C_{2} \theta^{2}} \\
\Psi(\theta) \approx & \frac{1}{8 C_{2}^{3 / 2}}\left(2 \sqrt{C_{2}}\left(C_{1}+2 C_{2} \theta\right) Q(\theta)+i\left(C_{1}^{2}-4 C_{2} W_{0}^{2}\right)\right. \\
& \left.\ln \left(-\frac{i\left(C_{1}+2 C_{2} \theta\right)}{\sqrt{C_{2}}}+2 Q(\theta)\right)\right) \\
J^{\prime}= & \frac{\partial H}{\partial \Psi} \\
J^{\prime}= & \frac{J}{Q} \sin (2 \Psi)\left(C_{3} e^{i \delta \theta}+C_{4} \theta e^{i \delta \theta}+C_{5} e^{-i \delta \theta}\right)
\end{aligned}
$$

The equation for the action can be approximated across the parametric resonance crossing by expanding $\Psi$ around the location of the parametric resonance $\theta_{r}$ and fixing $Q(\theta)=Q\left(\theta_{r}\right)$. And expansion out to second order for $\Psi$ captures its evolution across the resonance adequately and can be integrated analytically.

\section{APENDIX: TRANSFORMATION TO RICCATI 1ST ORDER DIFFERENTIAL EQUATION}

For fun we can transform this into a general 1st order Riccati nonlinear differential equation, using the following transformation,

$$
y=-\frac{\Psi_{I}^{+\prime} 2}{\Psi_{I}^{+} \xi}
$$

to get,

$$
y^{\prime}=y^{2} \frac{\xi}{2}+i f_{3} y-\frac{\xi^{*}}{2}
$$

While there are some non-linear approximate approaches to handle the Riccati Equation [6] the results will still require taking the integral in Eq. (57) to get back to Psi which will need to be performed numerically. 
[1] M. Froissart, and R. Stora, Nucl. Inst. Meth. 7, 297 (1960)

[2] S.Y.Lee "Accelerator Physics" World Scientific (1999) pp. 199-206.

[3] S. R. Mane, "Approximate formula for the asymptotic polarization for passage across two overlapping spin resonances," Nucl. Instrum. Meth. A 524, 80 (2004).

[4] L. NG, R. Rand and M. O'Neil Slow Passage Through Resonance in Mathieu's Equation (2002) http://www.math.cornell.edu/ rand/randpdf/slowpas2.pdf
J. Bridge, R. Rand, S. Sah, Slow Passage through Multiple Parametric Resonance Tongues. (2008) http://audiophile.tam.cornell.edu/randpdf/slowpas3.pdf [5] R. Baartman et. al. "Analysis of Rapid Betatron Resonance Crossing" Proc. Part. Acc. Conf. Knoxville, Tn (2005) pp 3206.

[6] Y. Tan and S. Abbasbandy, "Homotopy analysis method for quadratic Riccati differential equation", Com. in NonLinear Science and Numberical Simulation 13 (2008) 539546. 